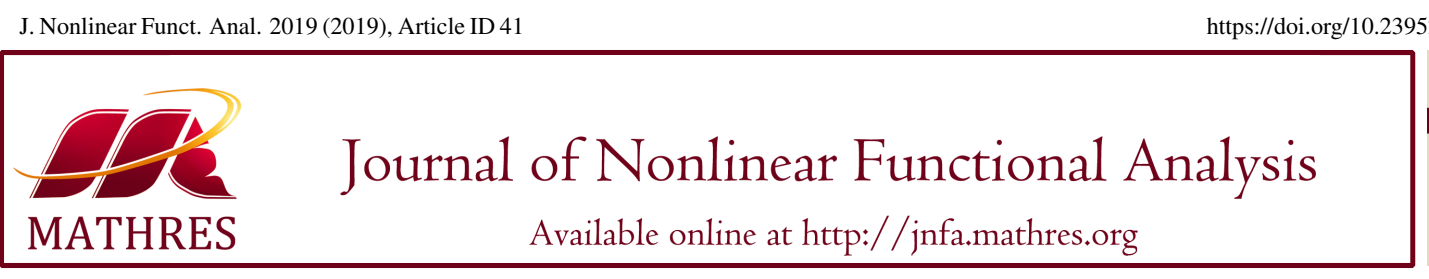

\title{
MULTIPLE NON-NEGATIVE SOLUTIONS OF A BOUNDARY VALUE PROBLEM OF THE FRACTIONAL THERMOSTAT MODEL
}

\author{
LIU YANG*, HUI ZHOU, CHUNFANG SHEN
}

College of Mathematics and Statistics, Hefei Normal University, Hefei 230061, Anhui, China

\begin{abstract}
In this paper, by means of the Avery-Peterson fixed point theorem, we establish the existence result of at least triple non-negative solutions of a boundary value problem of the fractional thermostat model which the derivative of unknown function is involved in the nonlinear term explicitly. An example illustrating our main result is given. Our results complements previous work in the area of the fractional thermostat model.

Keywords. Avery-Peterson fixed point theorem; Boundary value problem; Cone; Fixed point; Positive solution; Thermostat model.
\end{abstract}

2010 Mathematics Subject Classification. 26A33, 34B15.

\section{INTRODUCTION}

In this paper, we discuss the existence of non-negative solutions of the nonlinear boundary value problem

$$
\begin{gathered}
-{ }^{C} D^{\alpha} u(t)=f\left(t, u(t), u^{\prime}(t)\right), t \in[0,1], \\
u^{\prime}(0)=0, \beta^{C} D^{\alpha-1} u(1)+u(\eta)=0,
\end{gathered}
$$

where $\beta \Gamma(\alpha)-(1-\eta)^{\alpha-1}>0$ and $f \in C([0,1] \times[0, \infty) \times(\infty,+\infty),(0,+\infty)), \eta \in(0,1), \beta>0$. And we always assume that the following Lipschitz type conditions hold, for each $r_{1}, r_{2}>0$, there exist $L_{r}>0$ such that for all $s_{1}, s_{2} \in[0,1], t_{1}, t_{2} \in\left[0, r_{1}\right], v_{1}, v_{2} \in\left[0, r_{2}\right]$,

$$
(h)\left|f\left(s_{2}, t_{2}, v_{2}\right)-f\left(s_{1}, t_{1}, v_{1}\right)\right| \leq L_{r} \max \left\{\left|s_{2}-s_{1}\right|,\left|t_{2}-t_{1}\right|\left|v_{2}-v_{1}\right|\right\} .
$$

A function $u(t) \in C^{2}([0,1], R)$ is said to be a solution of (1.1)-(1.2) if $u(t)$ satisfies the equation (1.1) on $[0,1]$ and satisfies the boundary conditions (1.2).

Problem (1.1)-(1.2), which are models for a thermostat, came from the studies of stationary solutions for a one-dimensional heat equation, corresponding to a heated bar, with a controller at 1 adding or removing heat dependent on the temperature detected by a sensor at point $\eta$. The boundary condition

${ }^{*}$ Corresponding author.

E-mail addresses: xjiangfeng@163.com (L. Yang), zhouhui0309@126.com (H. Zhou), shencf2003@163.com (C. Shen).

Received November 19, 2018; Accepted September 9, 2019.

(C)2019 Journal of Nonlinear Functional Analysis 
(1.2) corresponds to the end at 0 being insulated. The original motivation of thermostat model came from the work by Guidotti and Merino [1] who studied the linear problem

$$
-u^{\prime \prime}(t)=y(t), t \in(0,1)
$$

with boundary conditions

$$
u^{\prime}(0)=0, u^{\prime}(1)+\beta u(0)=0, \beta>0,
$$

which models the stationary state of a heated bar of length 1 , insulated at $t=0$, with a controller adding or removing heat at $t=1$ depending on the temperature detected by a point sensor at $t=0$. Infante and Webb [2] considered the nonlinear problem

$$
\begin{gathered}
-u^{\prime \prime}(t)=f(t, u(t)), t \in(0,1), \\
u^{\prime}(0)=0, \beta u^{\prime}(1)+u(\eta)=0, \eta \in(0,1),
\end{gathered}
$$

where $\beta>0$ and $f$ is a non-negative function. The existence and uniqueness results of solutions were established by using the theory of fixed point index [3]. The authors also showed that solutions of problem (1.5)-(1.6) lose positivity as parameter $\beta$ decreases. Webb [4] discussed the existence of positive solutions of boundary value problem

$$
\begin{gathered}
-u^{\prime \prime}(t)=g(t) f(t, u(t)), t \in(0,1), \\
u^{\prime}(0)=\alpha[u], u^{\prime}(1)+\beta[u]=0,
\end{gathered}
$$

where $\alpha$ and $\beta$ are given by Riemann-Stieltjes integrals, which include the multi-point and integral types of boundary conditions as special cases. The author studied the useful properties of the Green's function and established the existence of positive solutions. For other results on thermostat models, we refer the readers to $[5,6,7,8,9,10]$ and the references therein. Most systems in practice have long-range temporal memory and the modelling of such systems by fractional-order differential equations has more advantages than classical models with integer-orders, see, e.g., $[11,12,13,14,15,16,17,18,19,20]$ and the references therein. Recently, Nieto and Pimentel [21] considered the following fractional analog of the thermostat model:

$$
\begin{gathered}
-{ }^{C} D^{\alpha}(t)=f(t, u(t)), t \in[0,1], \\
u^{\prime}(0)=0, \beta^{C} D^{\alpha-1} u(1)+u(\eta)=0,
\end{gathered}
$$

where $1<\alpha \leq 2$. The results in this paper extend the second-order thermostat model to the non-integer case.

We notice that in the results on thermostat model the existence results of positive solutions were all established under the assumption that the derivative of the unknown function $u(t)$ was not involved in the nonlinear term explicitly. On account of the physical meaning of $u^{\prime}(t)$, which describes the variety of the temperature of thermostat, it is of interest to consider the thermostat model in which the derivative of the unknown function $u(t)$ is involved in the nonlinear term explicitly. In this paper, we use the properties of the corresponding Green's functions and the Avery-Peterson fixed point theorem to show the existence of multiple non-negative solutions of problem (1.1)-(1.2). 


\section{PRELIMINARIES}

Definition 2.1. The Riemann-Liouville fractional integral of order $\alpha>0$ of a function $u(t)$ is given by

$$
I_{0+}^{\alpha} u(t)=\frac{1}{\Gamma(\alpha)} \int_{0}^{t}(t-s)^{\alpha-1} u(s) d s
$$

provided the integral exist.

Definition 2.2. The Caputo's fractional derivative of order $\alpha>0$ of a function $u(t)$ is given by

$$
{ }^{C} D_{0+}^{\alpha} u(t)=\frac{1}{\Gamma(n-\alpha)} \int_{0}^{t} \frac{u^{(n)}(s)}{(t-s)^{\alpha-n+1}} d s,(n-1<\alpha \leq n)
$$

where $n$ is the smallest integer greater than or equal to $\alpha$.

Lemma 2.3. Let $\alpha>0$. The fractional differential equation ${ }^{C} D_{0+}^{\alpha} u(t)=0$ has a solution

$$
u(t)=C_{1}+C_{2} t+C_{3} t^{2}+\ldots+C_{n} t^{n-1}, C_{i} \in R, i=1,2, \cdots, n .
$$

Lemma 2.4. Assume that $u(t) \in C^{n}[0,1] \cap L[0,1]$ with a fractional derivative of order $\alpha>0$ that belongs to $C[0,1] \cap L[0,1]$. Then

$$
I_{0+}^{\alpha}\left({ }^{C} D_{0+}^{\alpha} u(t)\right)=u(t)+C_{1}+C_{2} t+C_{3} t^{2}+\ldots+C_{n} t^{n-1}, C_{i} \in R, i=1,2, \cdots, n,
$$

where $n$ is the smallest integer greater than or equal to $\alpha$.

Definition 2.5. Let $E$ be a real Banach space. A nonempty convex closed set $P$ is called a cone provided that:

(1) $a u \in P$, for all $u \in P, a \geq 0$;

(2) $u,-u \in P$ implies $u=0$.

Definition 2.6. The map $\phi$ is said to be a nonnegative continuous convex functional on the cone $P$ of a real Banach space $E$ provided that $\phi: P \rightarrow[0,+\infty)$ is continuous and

$$
\phi(t x+(1-t) y) \leq t \phi(x)+(1-t) \phi(y), x, y \in P, t \in[0,1] .
$$

Definition 2.7. The map $\beta$ is said to be a nonnegative continuous concave functional on the cone $P$ of a real Banach space $E$ provided that $\beta: P \rightarrow[0,+\infty)$ is continuous and

$$
\beta(t x+(1-t) y) \geq t \beta(x)+(1-t) \beta(y), x, y \in P, t \in[0,1] .
$$

Let $\gamma, \theta$ be nonnegative continuous convex functionals on $P$. Let $\phi$ be a nonnegative continuous concave functional on $P$ and let $\psi$ be a nonnegative continuous functional on $P$. For positive numbers $a, b, c$ and $d$, we define the following convex sets:

$$
\begin{aligned}
& P(\gamma, d)=\{x \in P \mid \gamma(x)<d\}, \\
& P(\gamma, \phi, b, d)=\{x \in P \mid b \leq \phi(x), \gamma(x) \leq d\}, \\
& P(\gamma, \theta, \phi, b, c, d)=\{x \in P \mid b \leq \phi(x), \theta(x) \leq c, \gamma(x) \leq d\},
\end{aligned}
$$

and a closed set

$$
R(\gamma, \psi, a, d)=\{x \in P \mid a \leq \psi(x), \gamma(x) \leq d\}
$$


Lemma 2.8. [22] Let $P$ be a cone in Banach space E. Let $\gamma, \theta$ be nonnegative continuous convex functionals on $P$. Let $\phi$ be a nonnegative continuous concave functional on $P$, and let $\psi$ be a nonnegative continuous functional on $P$ satisfying

$$
\begin{gathered}
\psi(\lambda x) \leq \lambda \psi(x), \text { for } 0 \leq \lambda \leq 1, \\
\phi(x) \leq \psi(x),\|x\| \leq l \gamma(x) \text { for } x \in \overline{P(\gamma, d)},
\end{gathered}
$$

where $\overline{P(\gamma, d)}$ is the closure of the set $P(\gamma, d)$. Suppose that $T: \overline{P(\gamma, d)} \rightarrow \overline{P(\gamma, d)}$ is completely continuous and there exist positive numbers $a, b, c$ with $a<b$ such that

$\left(S_{1}\right)\{x \in P(\gamma, \theta, \phi, b, c, d) \mid \phi(x)>b\} \neq \emptyset$ and $\phi(T x)>b$ for $x \in P(\gamma, \theta, \phi, b, c, d)$;

$\left(S_{2}\right) \phi(T x)>b$ for $x \in P(\gamma, \phi, b, d)$ with $\theta(T x)>c$;

$\left(S_{3}\right) 0 \notin R(\gamma, \psi, a, d)$ and $\psi(T x)<$ a for $x \in R(\gamma, \psi, a, d)$ with $\psi(x)=a$.

Then $T$ has at least three fixed points $x_{1}, x_{2}, x_{3} \in \overline{P(\gamma, d)}$ such that:

$$
\gamma\left(x_{i}\right) \leq d, i=1,2,3 ; b<\phi\left(x_{1}\right) ; a<\psi\left(x_{2}\right), \phi\left(x_{2}\right)<b ; \psi\left(x_{3}\right)<a .
$$

\section{MAin Results}

Lemma 3.1. Suppose that $y(t) \in A C[0,1]$,

$$
w(t)=\int_{0}^{1} G(t, s) y(s) d s \in A C^{2}[0,1],
$$

then the following boundary value problem

$$
\begin{gathered}
{ }^{C} D_{0+}^{\alpha} u(t)+y(t)=0, t \in[0,1], \\
u^{\prime}(0)=0, \beta^{C} D^{\alpha-1} u(1)+u(\eta)=0
\end{gathered}
$$

is equivalent to

$$
u(t)=\int_{0}^{1} G(t, s) y(s) d s
$$

where

$$
G(t, s)= \begin{cases}\beta-\frac{(t-s)^{\alpha-1}}{\Gamma(\alpha)}+\frac{(\eta-s)^{\alpha-1}}{\Gamma(\alpha)}, & 0 \leq s \leq \eta, s \leq t, \\ \beta+\frac{(\eta-s)^{\alpha-1}}{\Gamma(\alpha)}, & 0 \leq s \leq \eta, s \geq t, \\ \beta-\frac{(t-s)^{\alpha-1}}{\Gamma(\alpha)}, & \eta \leq s \leq 1, s \leq t, \\ \beta, & \eta \leq s \leq 1, s \geq t .\end{cases}
$$

Proof. Suppose that $u(t)$ is a solution of problem (3.1-3.2), then from the definition and properties of the fractional derivative and integral, we have

$$
u(t)=-\int_{0}^{t} \frac{(t-s)^{\alpha-1}}{\Gamma(\alpha)} y(s) d s+c_{0}+c_{1} t
$$

Then

$$
u^{\prime}(t)=-\int_{0}^{t} \frac{(t-s)^{\alpha-2}}{\Gamma(\alpha-1)} y(s) d s+c_{1},
$$

which, together with the boundary condition $u^{\prime}(0)=0$, gives that $c_{1}=0$. Thus

$$
u(t)=-\int_{0}^{t} \frac{(t-s)^{\alpha-1}}{\Gamma(\alpha)} y(s) d s+c_{0} .
$$


From the fact $\beta^{C} D^{\alpha-1} u(1)+u(\eta)=0$, one has

$$
c_{0}=\beta \int_{0}^{1} y(s) d s+\int_{0}^{\eta} \frac{(\eta-s)^{\alpha-1}}{\Gamma(\alpha)} y(s) d s .
$$

It follows that

$$
u(t)=-\int_{0}^{t} \frac{(t-s)^{\alpha-1}}{\Gamma(\alpha)} y(s) d s+\beta \int_{0}^{1} y(s) d s+\int_{0}^{\eta} \frac{(\eta-s)^{\alpha-1}}{\Gamma(\alpha)} y(s) d s=\int_{0}^{1} G(t, s) y(s) d s .
$$

Conversely, we first see that $w^{\prime}(t)=-I^{\alpha-1} y(t)$. Then $w^{\prime \prime}(t)=-\left(I^{\alpha-1} y(t)\right)^{\prime}=-{ }^{C} D^{2-\alpha} y(t)$ for a.e. $t \in[0,1]$. Since $y \in A C[0,1]$ and $2-\alpha \in(0,1)$, we have ${ }^{C} D^{\alpha} w(t)=I^{2-\alpha} w^{\prime \prime}(t)=-y^{\prime \prime}(t)$ for a.e. $t \in[0,1]$ and (3.1) holds. Also we can check that boundary condition (3.2) holds.

Lemma 3.2. The function $G(t, s)$ satisfies the following conditions:

(1) $G(t, s) \in C([0,1] \times[0,1)), G(t, s)>0$, for $t, s \in(0,1)$;

(2) There exist a positive number $\gamma$ such that

$$
\min _{t, s \in[0,1]} G(t, s) \geq \gamma_{0} \max _{t, s \in[0,1]} G(t, s)
$$

where

$$
\gamma_{0}=\frac{\beta \Gamma(\alpha)-(1-\eta)^{\alpha-1}}{\beta \Gamma(\alpha)+\eta^{\alpha-1}}
$$

Proof. It is easy to check that $G(t, s)$ is continuous. Then, for each fixed $s \in[0,1]$, we have

$$
\frac{\partial G(t, s)}{\partial t}= \begin{cases}-\frac{(\alpha-1)}{\Gamma(\alpha)}(t-s)^{\alpha-2}, & s \leq t \\ 0, & s \geq t\end{cases}
$$

Then $G(t, s)$ is decreasing on $t$ for each fixed point $s \in[0,1]$. Thus

$$
\begin{gathered}
\min _{t, s \in[0,1]} G(t, s)=\frac{\beta \Gamma(\alpha)-(1-\eta)^{\alpha-1}}{\Gamma(\alpha)}, \\
\max _{t, s \in[0,1]} G(t, s)=\frac{\beta \Gamma(\alpha)+\eta^{\alpha-1}}{\Gamma(\alpha)}
\end{gathered}
$$

and

$$
\min _{t, s \in[0,1]} G(t, s) \geq \gamma_{0} \max _{t, s \in[0,1]} G(t, s)
$$

Lemma 3.3. Assume that $y(t)>0$ and $u(t)$ is a solution of problem (3.1)-(3.2). Then there exist a positive constant $\gamma_{1}$ such that

$$
\max _{0 \leq t \leq 1}\left|u^{\prime}(t)\right| \geq \gamma_{1} \max _{0 \leq t \leq 1}|u(t)|,
$$

where $\gamma_{1}=\frac{\Gamma(\alpha)}{\Gamma(\alpha-1)\left(\beta \Gamma(\alpha)+\eta^{\alpha-1}\right)}>0$. 
Proof. In view of Lemma 3.1, we obtain that

$$
u^{\prime}(t)=-\int_{0}^{t} \frac{(t-s)^{\alpha-2}}{\Gamma(\alpha-1)} y(s) d s
$$

and

$$
\begin{aligned}
\max _{0 \leq t \leq 1}\left|u^{\prime}(t)\right| & \geq\left|u^{\prime}(1)\right| \\
& =\int_{0}^{1} \frac{(1-s)^{\alpha-2}}{\Gamma(\alpha-1)} y(s) d s \\
& \geq \int_{0}^{1} \frac{1}{\Gamma(\alpha-1)} y(s) d s \geq \gamma_{1} \int_{0}^{1} G(t, s) y(s) d s .
\end{aligned}
$$

Thus,

$$
\max _{0 \leq t \leq 1}\left|u^{\prime}(t)\right| \geq \gamma_{1} \max _{0 \leq t \leq 1}|u(t)|, t \in[0,1] .
$$

Let the space $X=C^{1}[0,1]$ endowed with the norm

$$
\|u\|:=\max \left\{\max _{0 \leq t \leq 1}|u(t)|, \max _{0 \leq t \leq 1}\left|u^{\prime}(t)\right|\right\} .
$$

It is well known that $X$ is a Banach Space. Define the cone $K \subset X$ by

$$
K=\left\{u \in X: u(t) \geq 0, \min _{t \in[0,1]} u(t) \geq \gamma_{0} \max _{0 \leq t \leq 1} u(t)\right\} .
$$

Lemma 3.4. Let $T: K \rightarrow X$ be the operator defined by

$$
(T u)(t):=\int_{0}^{1} G(t, s) f\left(s, u(s), u^{\prime}(s)\right) d s .
$$

Then $T: K \rightarrow K$ is completely continuous and satisfies

$$
\max _{0 \leq t \leq 1}\left|(T u)^{\prime}(t)\right| \geq \gamma_{1} \max _{0 \leq t \leq 1}|T u(t)| .
$$

Proof. In view of the continuity of function $G(t, s)$ and $f\left(t, u(t), u^{\prime}(t)\right), T: K \rightarrow X$ is continuous. Let $\Omega \subset K$ be bounded. Then there exist a positive constant $R_{1}>0$ such that $\|u\| \leq R_{1}, u \in \Omega$. Denote

$$
R=\max _{0 \leq t \leq 1, u \in \Omega}\left|f\left(t, u(t), u^{\prime}(t)\right)\right|+1
$$

For $u \in \Omega$, we have

$$
|T u| \leq \int_{0}^{1} G(t, s)\left|f\left(s, u(s), u^{\prime}(s)\right)\right| d s \leq \frac{\beta \Gamma(\alpha)+\eta^{\alpha-1}}{\Gamma(\alpha)} R
$$

and

$$
\begin{aligned}
\left|(T u)^{\prime}(t)\right| & =\left|-\frac{1}{\Gamma(\alpha-1)} \int_{0}^{t}(t-s)^{\alpha-2} f\left(s, u(s), u^{\prime}(s)\right) d s\right| \\
& \leq \frac{R}{\Gamma(\alpha-1)} \int_{0}^{1}(1-s)^{\alpha-2} d s \\
& =\frac{R}{(\alpha-1) \Gamma(\alpha-1)}=\frac{R}{\Gamma(\alpha)} .
\end{aligned}
$$


Hence $T(\Omega)$ is bounded. For $u \in \Omega, t_{1}, t_{2} \in[0,1]$, one has

$$
\begin{aligned}
& \left|T u\left(t_{2}\right)-T u\left(t_{1}\right)\right| \\
& \left.=\frac{1}{\Gamma(\alpha)} \mid \int_{0}^{t_{2}}\left(t_{2}-s\right)^{\alpha-1} f\left(s, u, u^{\prime}(s)\right) d s-\int_{0}^{t_{1}}\left(t_{1}-s\right)^{\alpha-1} f\left(s, u, u^{\prime}(s)\right) d s\right) \mid \\
& \leq \frac{R}{\Gamma(\alpha+1)}\left|t_{2}^{\alpha}-t_{1}^{\alpha}\right|
\end{aligned}
$$

and

$$
\begin{aligned}
& \left|(T u)^{\prime}\left(t_{2}\right)-(T u)^{\prime}\left(t_{1}\right)\right| \\
& \leq\left|\frac{1}{\Gamma(\alpha-1)}\left(\int_{0}^{t_{2}}\left(t_{1}-s\right)^{\alpha-2} f\left(s, u, u^{\prime}(s)\right) d s-\int_{0}^{t_{1}}\left(t_{2}-s\right)^{\alpha-2} f\left(s, u, u^{\prime}(s)\right) d s\right)\right| \\
& =\frac{1}{\Gamma(\alpha-1)}\left|\int_{0}^{t_{1}}\left(\left(t_{2}-s\right)^{\alpha-2}-\left(t_{1}-s\right)^{\alpha-2}\right) f\left(s, u(s), u^{\prime}(s)\right) d s+\int_{t_{1}}^{t_{2}}\left(t_{2}-s\right)^{\alpha-2} f\left(s, u(s), u^{\prime}(s)\right) d s\right| \\
& \leq \frac{R}{\Gamma(\alpha)}\left(\left|t_{2}^{\alpha-1}-t_{1}^{\alpha-1}\right|+2\left|t_{2}-t_{1}\right|^{\alpha-1}\right) .
\end{aligned}
$$

Thus,

$$
\left\|T u\left(t_{2}\right)-T u\left(t_{1}\right)\right\| \rightarrow 0 \text { for } t_{1} \rightarrow t_{2}, u \in \Omega .
$$

By means of the Arzela-Ascoli theorem, we claim that $T$ is completely continuous. Finally, we see that

$$
\begin{aligned}
\min _{0 \leq t \leq 1}|T u(t)| & =\min _{0 \leq t \leq 1} \int_{0}^{1} G(t, s) f\left(s, u(s), u^{\prime}(s)\right) d s \\
& \geq \gamma_{0} \max _{0 \leq t \leq 1} \int_{0}^{1} G(t, s) f\left(s, u(s), u^{\prime}(s)\right) d s \\
& =\gamma_{0} \max _{0 \leq t \leq 1}(T u)(t),
\end{aligned}
$$

and

$$
\begin{aligned}
\max _{0 \leq t \leq 1}\left|T u^{\prime}(t)\right| & \geq \int_{0}^{1} \frac{(1-s)^{\alpha-2}}{\Gamma(\alpha-1)} f\left(s, u(s), u^{\prime}(s)\right) d s d s \\
& \geq \int_{0}^{1} \frac{1}{\Gamma(\alpha-1)} f\left(s, u(s), u^{\prime}(s)\right) d s d s \\
& \geq \gamma_{1} \int_{0}^{1} G(t, s) f\left(s, u(s), u^{\prime}(s)\right) d s d s \\
& \geq \gamma_{1} \max _{0 \leq t \leq 1}|T u(t)| .
\end{aligned}
$$

Thus, we show that $T: K \rightarrow K$ and satisfies that

$$
\max _{0 \leq t \leq 1}\left|(T u)^{\prime}(t)\right| \geq \gamma_{1} \max _{0 \leq t \leq 1}|T u(t)| .
$$

Let the nonnegative continuous concave functional $\alpha$, the nonnegative continuous convex functionals $\gamma$, and $\theta$, and the nonnegative continuous functional $\psi$ be defined on the cone by

$$
\gamma(u)=\max _{0 \leq t \leq 1}\left|u^{\prime}(t)\right|, \theta(u)=\psi(u)=\max _{0 \leq t \leq 1}|u(t)|, \phi(u)=\min _{0 \leq t \leq 1}|u(t)| .
$$

By Lemmas 3.3 and 3.4, the functionals defined above satisfy that

$$
\gamma_{0} \theta(u) \leq \phi(u) \leq \theta(u)=\psi(u),\|u\| \leq \gamma_{2} \gamma(u), u \in K,
$$


where $\gamma_{2}=\max \left\{\frac{1}{\gamma_{1}}, 1\right\}$. Therefore, the condition (2.6) of Lemma 2.3 is satisfied. Assume that there exist constants $0<a, b, d$ with $a<b<d, c=\frac{b}{\gamma_{0}}$ and $\left(\beta \Gamma(\alpha)-(1-\eta)^{\alpha-1}\right) d>b$ such that

$\left(A_{1}\right) f(t, u, v) \leq \Gamma(\alpha) d, \quad \forall(t, u, v) \in[0,1] \times\left[0, \gamma_{2} d\right] \times[-d, d] ;$

$\left(A_{2}\right) f(t, u, v)>\frac{\Gamma(\alpha)}{\beta \Gamma(\alpha)-(1-\eta)^{\alpha-1}} b, \quad \forall(t, u, v) \in[0,1] \times\left[b, b / \gamma_{0}\right] \times[-d, d] ;$

$\left(A_{3}\right) f(t, u, v)<\frac{\Gamma(\alpha)}{\beta \Gamma(\alpha)+\eta^{\alpha-1}} a, \quad \forall(t, u, v) \in[0,1] \times[0, a] \times[-d, d]$.

Theorem 3.5. Under assumptions $\left(A_{1}\right)-\left(A_{3}\right)$, problem (1.1)-(1.2) has at least three non-negative solutions $u_{1}, u_{2}, u_{3}$ satisfying

$$
\max _{0 \leq t \leq 1}\left|u_{i}^{\prime}(t)\right| \leq d, i=1,2,3 ; b<\min _{0 \leq t \leq 1}\left|u_{1}(t)\right| ; a<\max _{0 \leq t \leq 1}\left|u_{2}(t)\right|, \min _{0 \leq t \leq 1}\left|u_{2}(t)\right|<b, \max _{0 \leq t \leq 1}\left|u_{3}(t)\right| \leq a
$$

Proof. Under the condition $h$, if $u(t)$ is a solution of the operator equation

$$
w(t)=\int_{0}^{1} G(t, s) f\left(s, u(s), u^{\prime}(s)\right) d s=(T u)(t) .
$$

then $w(t) \in A C^{2}[0,1]$ (see Proposition 3.1 in [23]). Problem (1.1)-(1.2) has a solution $u=u(t)$ if and only if $u$ solves the operator equation (3.14).For $u \in \overline{K(\gamma, d)}$, we have $\gamma(u)=\max _{0 \leq t \leq 1}\left|u^{\prime}(t)\right|<d$. From assumption $\left(A_{1}\right)$, we obtain $f\left(t, u(t), u^{\prime}(t)\right) \leq \Gamma(\alpha) d$. Thus

$$
\begin{aligned}
\gamma(T u) & =\max _{0 \leq t \leq 1}\left|-\frac{1}{\Gamma(\alpha-1)} \int_{0}^{t}(t-s)^{\alpha-2} f\left(s, u(s), u^{\prime}(t)\right) d s\right| \\
& \leq \frac{1}{\Gamma(\alpha-1)} \times \frac{1}{\alpha-1} \times \Gamma(\alpha) d \\
& =d .
\end{aligned}
$$

Hence, $T: \overline{K(\gamma, d)} \rightarrow \overline{K(\gamma, d)}$. The fact that the constant function $u(t)=\frac{b}{\gamma_{0}} \in K(\gamma, \theta, \phi, b, c, d)$ and $\phi\left(\frac{b}{\gamma_{0}}\right)>b$ implies that $\{u \in K(\gamma, \theta, \phi, b, c, d \mid \phi(u)>b)\} \neq \emptyset$. For $u \in K(\gamma, \theta, \phi, b, c, d)$, we have $b \leq u(t) \leq \frac{b}{\gamma_{0}}$ and $\left|u^{\prime}(t)\right|<d$ for $0 \leq t \leq 1$. From assumption $\left(A_{2}\right)$, we see

$$
f\left(t, u(t), u^{\prime}(t)\right)>\frac{\Gamma(\alpha)}{\beta \Gamma(\alpha)-(1-\eta)^{\alpha-1}} b
$$

Thus

$$
\begin{aligned}
\phi(T u) & =\min _{0 \leq t \leq 1}\left|\int_{0}^{1} G(t, s) f\left(s, u(s), u^{\prime}(s)\right) d s\right| \\
& \geq \frac{\beta \Gamma(\alpha)-(1-\eta)^{\alpha-1}}{\Gamma(\alpha)} \times \frac{\Gamma(\alpha)}{\beta \Gamma(\alpha)-(1-\eta)^{\alpha-1}} b=b,
\end{aligned}
$$

which means

$$
\phi(T u)>b, \forall u \in K\left(\gamma, \theta, \phi, b, \frac{b}{\gamma_{0}}, d\right) .
$$

These ensure that the condition ( $S 1)$ of Lemma 2.8 is satisfied. For all $u \in K(\gamma, \phi, b, d)$ with $\theta(T u)>c$,

$$
\phi(T u) \geq \gamma_{0} \theta(T u)>\gamma_{0} c=\gamma_{0} \frac{b}{\gamma_{0}}=b .
$$


Thus, the condition $\left(S_{2}\right)$ of Lemma 2.8 holds. Finally, we show that $\left(S_{3}\right)$ also holds. We see that $\psi(0)=0<a$ and $0 \notin R(\gamma, \psi, a, d)$. Suppose that $u \in R(\gamma, \psi, a, d)$ with $\psi(x)=a$. Using assumption $\left(A_{3}\right)$, one has

$$
\begin{aligned}
\psi(T u) & =\max _{0 \leq t \leq 1}\left|\int_{0}^{1} G(t, s) f\left(s, u(s), u^{\prime}(s)\right) d s\right| \\
& \leq \frac{\beta \Gamma(\alpha)+\eta^{\alpha-1}}{\Gamma(\alpha)} \times \frac{\Gamma(\alpha)}{\beta \Gamma(\alpha)+\eta^{\alpha-1}} a \\
& =a .
\end{aligned}
$$

Thus, all the conditions of Lemma 2.8 are satisfied. Hence problem (1.1)-(1.2) has at least three nonnegative solutions $u_{1}, u_{2}, u_{3}$ satisfying

$$
\max _{0 \leq t \leq 1}\left|u_{i}^{\prime}(t)\right| \leq d, i=1,2,3 ; b<\min _{0 \leq t \leq 1}\left|u_{1}(t)\right| ; a<\max _{0 \leq t \leq 1}\left|u_{2}(t)\right|, \min _{0 \leq t \leq 1}\left|u_{2}(t)\right|<b, \max _{0 \leq t \leq 1}\left|u_{3}(t)\right| \leq a .
$$

\section{The EXAMPLE}

In this section, we present an example to illustrate the main theorems. Consider the nonlinear boundary value problem

$$
\begin{gathered}
D_{0+}^{1.7} u(t)+f\left(t, u(t), u^{\prime}(t)\right)=0, t \in(0,1), \\
u^{\prime}(0)=0, \frac{2}{3} D_{0+}^{0.7} u(1)+u\left(\frac{3}{4}\right)=0,
\end{gathered}
$$

where $\alpha=1.7, \beta=\frac{2}{3}, \eta=\frac{3}{4}$ and

$$
f(t, u, v)= \begin{cases}\frac{1}{\pi^{2}} e^{t}+\frac{1}{4} u^{3}+\frac{1}{100} \sin \left(\frac{v}{1000}\right), & 0 \leq u \leq 12, \\ \frac{1}{\pi^{2}} e^{t}+432+\frac{1}{100} \sin \left(\frac{v}{1000}\right), & u>12\end{cases}
$$

By a straightforward calculation, we see that

$$
\begin{gathered}
\gamma_{0}=\frac{\beta \Gamma(\alpha)-(1-\eta)^{\alpha-1}}{\beta \Gamma(\alpha)+\eta^{\alpha-1}}=0.1594 \\
\gamma_{1}=\frac{\Gamma(\alpha)}{\Gamma(\alpha-1)\left(\beta \Gamma(\alpha)+\eta^{\alpha-1}\right)}=0.4918
\end{gathered}
$$

and

$$
\gamma_{2}=\max \left\{\frac{1}{\gamma_{1}}, 1\right\}=2.0333
$$

We choose positive constants $a=1, b=5$ and $d=1000$, and check that the nonlinear term $f(t, u, v)$ satisfies

(1) $f(t, u, v)<\Gamma(\alpha) d \approx 908.6$ $(t, u, v) \in[0,1] \times[0,2034] \times[-1000,1000] ;$

(2) $f(t, u, v)>\frac{\Gamma(\alpha)}{\beta \Gamma(\alpha)-(1-\eta)^{\alpha-1}} b \approx 20.0291,(t, u, v) \in[0,1] \times[5,31.3676] \times[-1000,1000]$;

(3) $f(t, u, v)<\frac{\Gamma(\alpha)}{\beta \Gamma(\alpha)+\eta^{\alpha-1}} a \approx 0.6384$, $(t, u, v) \in[0,1] \times[0,1] \times[-1000,1000]$. 
Then all the assumptions of Theorem 3.5 are satisfied. Thus problem (4.1)-(4.2) has at least three nonnegative solutions $u_{1}(t), u_{2}(t), u_{3}(t)$ satisfying

$\max _{0 \leq t \leq 1}\left|u_{i}^{\prime}(t)\right| \leq 1000, i=1,2,3 ; 5<\min _{0 \leq t \leq 1}\left|u_{1}(t)\right|, 1<\max _{0 \leq t \leq 1}\left|u_{2}(t)\right|, \min _{0 \leq t \leq 1}\left|u_{2}(t)\right|<5, \max _{0 \leq t \leq 1}\left|u_{3}(t)\right| \leq 1$.

Remark 4.1. We see that the first order derivative of function $u(t)$ is involved in the nonlinear term of problem (4.1)-(4.2) explicitly. The previous results for positive solutions of fractional thermostat model are not applicable to this problem.

\section{Funding}

This paper was sponsored by the NSFC (11201109), Anhui Provincial Natural Science Foundation (1708085MA16), and the Higher School Natural Science Project of Anhui Province(KJ2017A937).

\section{REFERENCES}

[1] P. Guidotti, S. Merino Gradual loss of positivity and hidden invariant cones in a scalar heat equation, Differential Integral Equations, 13 (2000), 1551-1568.

[2] G. Infante, J.R.L. Webb, Loss of positivity in a nonlinear scalar heat equation, Nonlinear Differential Equations Appl. 13 (2006) 249-261.

[3] D. Guo, V. Lakshmikantham, Nonlinear Problems in Abstract Cones, Academic Press, New York, 1988

[4] J.R.L. Webb, Existence of positive solutions for a thermostat model, Nonlinear Anal. 13 (2012), 923-938.

[5] P. Palamides, G. Infante, P. Pietramala, Nontrival solutions of a nonlinear heat flow problem via Sperner's Lemma, Appl. Math. Lett. 22 (2009), 1444-1450.

[6] G. Infante, Nonlocal boundary value problems with two nonlinear boundary conditions, Commum. Appl. Anal. 12 (2008), 279-288.

[7] G. Infante, J.R.L. Webb, Nonlinear Non-local boundary-value problems and perturbed Hammerstein integral equations, Proc. Edinb. Math. Soc. 49 (2006), 637-656.

[8] J.R.L. Webb, Optimal constants in a nonlocal boundary value problem, Nonlinear Anal. 63 (2005), 672-685.

[9] C. Shen, H. Zhou, L. Yang, Existence and nonexistence of positive solutions of a fractional thermostat model with a parameter, Math. Methods Appl. Sci. 39 (2016), 4504-4511.

[10] A. Calamai, G. Infante, Nontrivial solutions of boundary value problems for second-order functional differential equations, Annali di Matematica, 195 (2016), 741-756.

[11] D. Delbosco, Fractional calculus and function spaces, J. Fract. Calc. 6 (1994), 45-53.

[12] K. S. Miller, B. Ross, An Introduction to the Fractional Calculus and Fractional Differential Equations, Wiley, New York, 1993.

[13] V. Lakshmikantham, J. Devi, Theory of fractional differential equations in a Banach space, European J. Pure. Appl. Math. 1 (2008), 38-45.

[14] V. Lakshmikantham, S. Leela, Nagumo-type uniqueness result for fractional differential equations, Nonlinear Anal. 71 (2009), 2886-2889.

[15] V. Lakshmikantham, S. Leela, A Krasnoselskii-Krein-type uniqueness result for fractional differential equations, Nonlinear Anal. 71 (2009), 3421-3424.

[16] V. Lakshmikantham, Theory of fractional differential equations, Nonlinear Anal. 69 (2008), 3337-3343.

[17] R.P. Agarwal, D. O'Regan, S. Stanek, Positive solutions for Dirichlet problems of singular nonlinear fractional differential equations, J. Math. Anal. Appl. 371 (2010), 57-68.

[18] D. Jiang, C. Yuan, The positive properties of the Green function for Direchlet-type boundary value problems of nonlinear fractional differential equations and its application, Nonlinear Anal. 15 (2010), 710-719.

[19] B. Ahmad, J. Nieto, Existence results for a coupled system of nonlinear fractional differential equations with three-point boundary conditions, Comput. Math. Appl. 58 (2009), 1838-1843.

[20] Z. Bai, On positive solutions of a nonlocal fractional boundary value problem, Nonlinear Anal. 72 (2010), 916-924. 
[21] J. Nieto, J. Pimentel, Positive solutions of a fractional thermostat model, Boundary Value Probl. 2013 (2013), Article ID 5.

[22] R.I. Avery, A.C. Peterson, Three positive fixed points of nonlinear operators on ordered Banach spaces, Comput. Math. Appl. 42 (2001), 313-322.

[23] K.Q. Lan, W. Lin, Positive solutions of systems of Caputo fractional differential equations, Commun. Appl. Anal. 17 (2013), 61-86. 
Addendum posted by the editor on August 26, 2020

The main results presented in this paper are not correct. The authors were sent a detailed report and were invited to make corrections but have not done so. The main difficulty is that solutions of the integral equation are $C^{1}$ functions but are not $C^{2}$ functions except in special cases, see Theorem 6.26 of K. Diethelm, The analysis of fractional differential equations. An application-oriented exposition using differential operators of Caputo type. Lecture Notes in Mathematics No. 2004. Springer-Verlag, Berlin, 2010. The definition of the Caputo differential operator from Diethelm's book should be used and not the Definition 2.2 of this paper. 